\title{
Endomyocardial Fibrosis in Infancy
}

\author{
Marcelo Biscegli Jatene, Ivan Salvador Bonillo Contreras, Laura C. Riera Lameda, \\ José de Lima Oliveira Jr., Munir Ebaid, Elisa Rumiko Iwahashi, Deipara Monteiro Abellan, \\ Vera Demarchi Aiello, Miguel Barbero Marcial, Sérgio Almeida de Oliveira
}

São Paulo, SP - Brazil

The patient was a 4-month-old infant, who underwent persistent ductus arteriosus interruption with titanium clips at the age of 13 days and, since the age of 2 months, had crises of hypoxia and hypertonicity. After clinical investigation, the presence of pulmonary hypertension was confirmed and left ventricular inflow tract obstruction was suspected. The patient underwent surgical treatment at the age of 4 months, during which right and left ventricular endocardial fibrosis was identified. The fibrosis was resected, but the infant had an unfavorable clinical evolution with significant diastolic restriction and died on the sixth postoperative day. Anatomicopathological and surgical findings suggested endomyocardial fibrosis, although that pathology is very rare at the patient's age.

Endomyocardial fibrosis was first reported by Davies and Ball in $1948^{1,2}$ as an endemic cardiac disease present in several tropical countries of the African and American continents. Some cases were also reported in Asia, North America, and Europe ${ }^{3}$.

Endomyocardial fibrosis is characterized by the formation of fibrous tissue in the endocardium. It may affect both sexes, has no ethnical differentiation, but is more common in young adults. Although the disease may affect both ventricles, isolated or not, the right ventricle is more frequently involved ${ }^{3}$.

Endocardial fibrosis may be present in the entire ventricular cavity, being usually more marked in the apical region and right ventricular inflow tract. When present in the apex, it may intensely impair ventricular function because of the reduction in the ventricular cavity and in diastolic relaxation.

Instituto do Coração of the Hospital das Clínicas of the FMUSP.

Mailing address: Marcelo Biscegli Jatene - InCor - Divisão Cirúrgica - Setor de Cirurgia Cardíaca Pediátrica - Av. Dr. Enéas C. Aguiar, 44 - 05403-000 - São Paulo, SP, Brazil - E-mail - mbjatene@uol.com.br

English version by Stela Maris C. e Gandour
Clinical repercussion is more significant when involvement of the subvalvular region and of the papillary muscles occurs, resulting in obstruction of adequate ventricular filling with variable symptoms of left or right heart failure, depending on the cavity involved ${ }^{3,4}$.

In an attempt to identify causal agents, the following factors have been suggested: environmental (nutritional deficiencies, use of toxic substances to the heart), ethnic, genetic, and immunological factors ${ }^{3,5}$; its etiology, however, still remains unknown.

Reports on endomyocardial fibrosis in pediatric patients are not common. It is more frequent from the fourth year of age on, with few reports in the first 2 years of life e $^{5-10}$.

This study aimed at reporting endomyocardial fibrosis in a 4-month-old infant, in addition to discussing aspects related to the clinical evolution and probable etiology of the disease.

\section{Case report}

The patient was a 4-month-old white male born from an elective Cesarean delivery of a first full-term pregnancy at the gestational age of 37 weeks and 3/7. The newborn weighed 3,400 $\mathrm{g}$ and had progressive respiratory distress right after birth. The mother took sintroid for hypothyroidism, and, because of Rh incompatibility (the mother was Rh-), she had taken gestadione since the fourth month of pregnancy. The newborn required orotracheal intubation in the sixth hour of life, when pneumonia was diagnosed, and he remained under mechanical ventilation for 7 days.

After extubation, the patient continued with mild respiratory distress and congestive lungs. The diagnosis of persistent ductus arteriosus and pulmonary hypertension was established. At the age of 13 days, he underwent persistent ductus arteriosus interruption with titanium clips through minithoracotomy, had a good evolution, and was discharged on the tenth postoperative day receiving digoxin and furosemide.

At the age of $2 \frac{1}{2}$ months, the patient was hospitalized 
due to a crisis of hypoxia with severe cyanosis, followed by hypertonicity and apnea. After stabilization, the patient underwent a neurological investigation, which resulted within the normal range; a genetic study, which was normal; and a new cardiological evaluation, which showed the following findings: pulmonary hypertension with systolic pulmonary pressure of $65 \mathrm{~mm} \mathrm{Hg}$, small atrial septal defect, and good ventricular function. The patient received nifedipine in association with other medications and improved slightly, with a reduction in the pulmonary pressure, but the cyanotic episodes persisted.

A new echocardiogram showed systolic pulmonary pressure of $70 \mathrm{mmHg}$, peak and mean mitral transvalvular gradients of $7 \mathrm{mmHg}$ and $2 \mathrm{mmHg}$, respectively, with shortening of the tendinous cords of the mitral valve, and no left ventricular dysfunction. Cardiac catheterization was performed and showed effective clipping of the ductus arteriosus, significant pulmonary hypertension, dilation of the left atrium and pulmonary veins, in addition to an image suggestive of mitral stenosis with amputation of the left ventricular tip (fig. 1).

The patient was referred for surgery, during which the following was observed: a normal appearing mitral valve with short tendinous cords supported by a fibrous structure, which obstructed the left ventricular inflow tract. Left ventricular tumor or endomyocardial fibrosis was suspected. A frozen biopsy showed fibrosis and no neoplastic cell.

A ventriculotomy was performed at the apex of the left ventricle, which was very hypertrophic due to the presence of a fibrous cover occupying the entire left ventricular cavity and encompassing the papillary muscles. A wide resection was performed, and fibrous tissue was left only on the papillary muscles. This alleviated the cavity, which was apparently small and restrictive. The right ventricle was dilated and affected by a lesser-degree fibrosis, which was also resected.

Extracorporeal circulation was withdrawn with the help of vasoactive drugs (dobutamine and milrinone) and of an epicardial pacemaker for total atrioventricular block. The systolic cavitary pressures after extracorporeal circu-

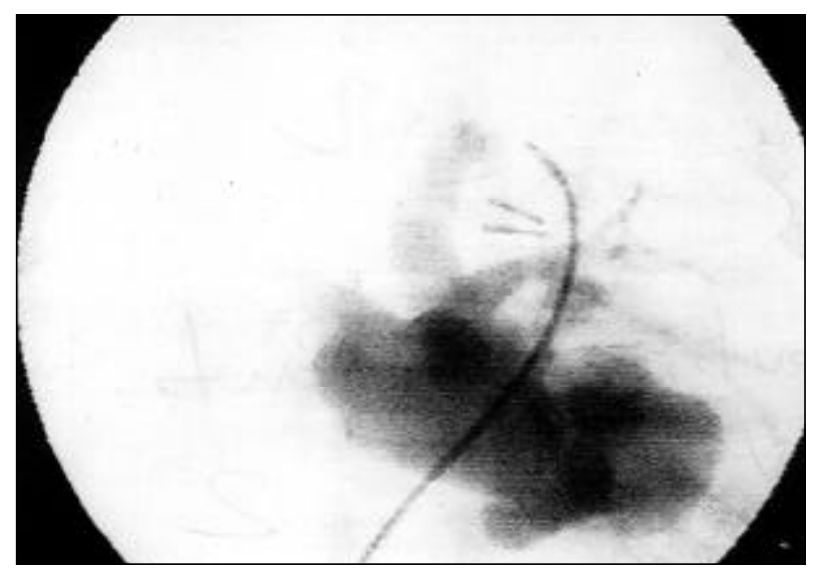

Fig. 1 - Left ventriculography showing amputation of the tip of the left ventricle. lation were as follows: right ventricle $-66 \mathrm{mmHg}$; left ventricle $-70 \mathrm{mmHg}$; pulmonary trunk $-64 \mathrm{mmHg}$; and left atrium $-18 \mathrm{mmHg}$.

The postoperative echocardiogram revealed the following: hypertrophic and dilated right ventricle with mild contractile deficit; left ventricle with good systolic function and important diastolic restriction; no pericardial effusion; mild mitral reflux; and systolic pulmonary pressure of $40 \mathrm{mmHg}$.

The patient evolved in the postoperative period with significant pulmonary hypertension, low cardiac output, renal failure, which required peritoneal dialysis, and reversed cardiopulmonary arrest on the first postoperative day. This evolution was complicated with hemodynamic instability and a new cardiopulmonary arrest on the sixth postoperative day, which did not respond to the resuscitation maneuvers, and the patient died.

The autopsy revealed an enlarged heart, with moderate dilation of both atria, and no congenital defects. Some apical thrombi were observed in the right ventricle, and, in the left ventricular inflow tract and apex, extensive raw myocardial areas were observed, corresponding to the previous wide surgical resection already cited.

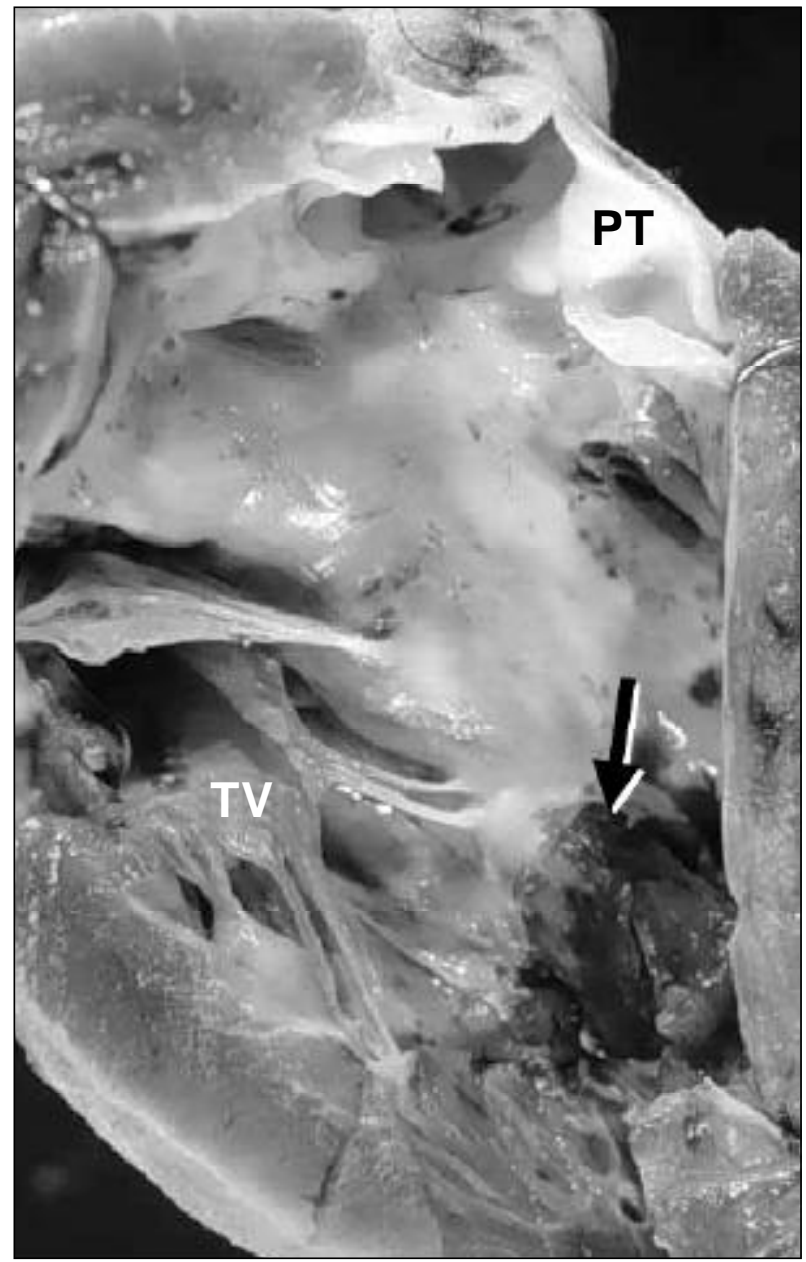

Fig. 2 - Opened right ventricle; presence of endocardial fibrosis involving the inflow tract close to the tricuspid valve (TV) and the outflow tract. Recent thrombi are observed in the apex. PT - pulmonary trunk. 


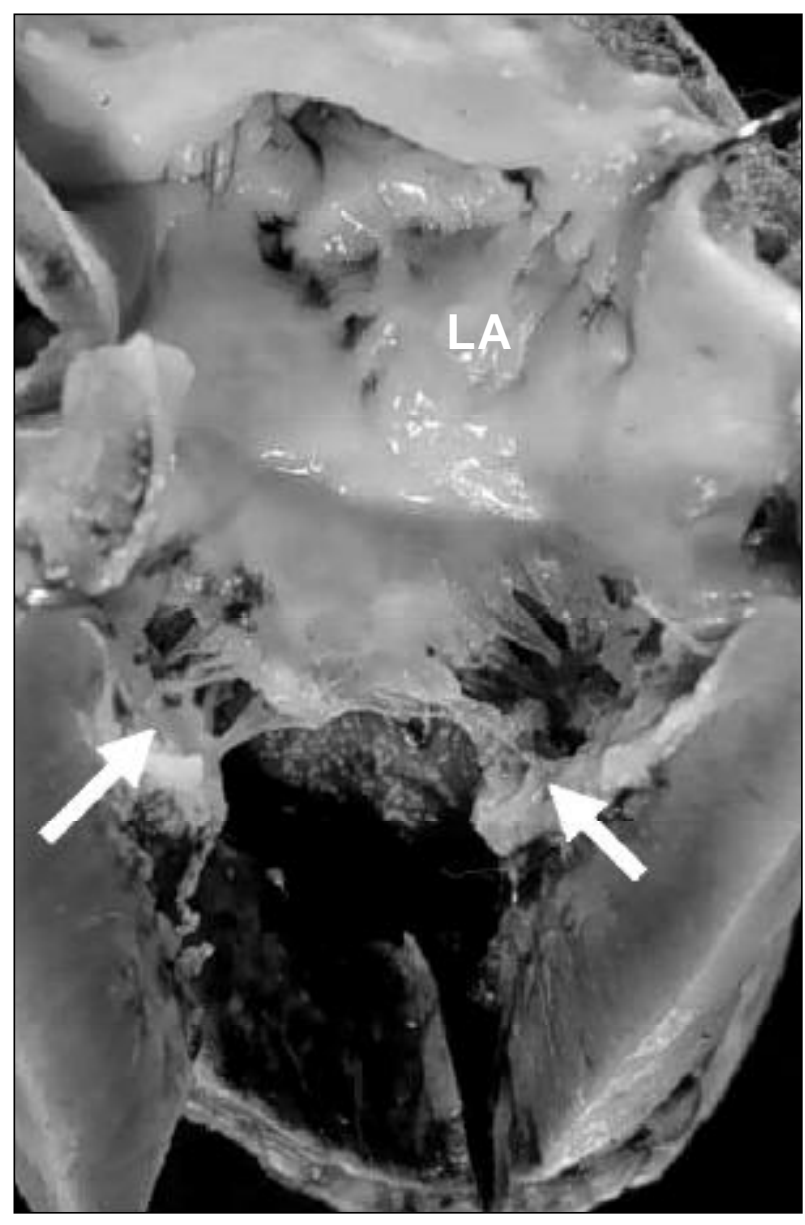

Fig. 3 - Opened left atrium and ventricle. The left ventricular wall is hypertrophic. Note the raw appearance of the endocardial lining with residual fibrosis involving the papillary muscles (arrows).

The remaining endocardium of both ventricles was thickened, mainly in the inflow tract areas. Shortening of the tendinous cords of the atrioventricular valves was observed, some of which were encompassed by fibrosis (figs. 2 and 3). The apical trabeculae were obliterated by dense fibrosis, which extended towards the myocardium in several points. Microscopically, endocardial thickening was

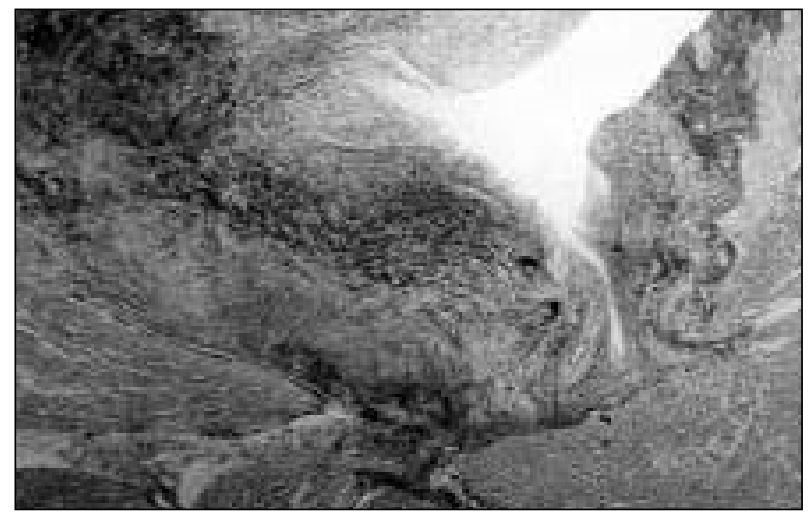

Fig. 4 - Microphotograph of the endocardium and myocardium showing fibrotic bundles (red areas) that penetrate towards the myocardium. The elastic fibers (in black) are increased and disorderly arranged. Verhoeff stain, original magnification 25X. present and characterized by dense fibrosis and disordered proliferation of elastic fibers (fig. 4). In addition, several neoformed vessels and nonspecific chronic inflammatory infiltration could be seen.

The histological study of the lungs showed obstructive lesions in the pulmonary arteries (II degree according to the Heath-Edwards criteria) and veins, compatible with passive pulmonary hypertension.

\section{Discussion}

Endomyocardial fibrosis is an endocardial affection of unknown etiopathogeny, originally described in Central Africa. In Brazil, similar cases have been reported, mainly in the populations with ethnic and socioeconomic characteristics similar to those found in the African population ${ }^{3}$. We report the case of a 4-month-old white male with no ethnic similarity to those reported in the literature.

Although the disease is more frequent in young adults, it may affect children above the age of 4 years ${ }^{5-7}$. We report the case of an infant who, since his first days of life, had symptoms suggestive of pulmonary hypertension, which, in the sequence of investigation and evolution, gave us the impression of being caused by endomyocardial fibrosis, which was definitively diagnosed during surgery. We suggested that the infant had the disease since birth and progressively developed symptoms, but the cardiac lesion was already installed.

The relation between endomyocardial fibrosis and the chronic use of anabolic steroids in adults has been reported, although not in children ${ }^{8}$. In our case, the use of steroids by the mother during the prenatal period is worth noting, although she used them for only a short period of time.

From the clinical point of view, the diastolic restriction of the ventricle affected by endomyocardial fibrosis more commonly leads to symptoms of heart failure and pulmonary hypertension. The magnitude of symptoms relates primarily to the degree of ventricular impairment, and, secondarily, to valvular impairment, which usually results from the inclusion of the papillary muscles and the subvalvular apparatus in the lesion of endomyocardial fibrosis. In our patient, the symptoms appeared early, and, when endomyocardial fibrosis was surgically suspected, restriction to the adequate ventricular filling due to mitral subvalvular stenosis already existed, but no ventricular dysfunction could be detected on the echocardiogram.

From our point of view, the infant already had an advanced degree of ventricular impairment (diastolic restriction) since birth, because of the early age at which the symptoms appeared.

Evidence suggests the participation of eosinophils in the pathogenesis of endomyocardial fibrosis; endocardial fibrosis has been reported in patients with increased eosinophilia of several causes ${ }^{9}$. An increase in eosinophils was also observed in our patient ( $18 \%$ or $1422 / \mathrm{mm}^{3}$ ), strengthening the suspicion of endomyocardial fibrosis.

The echocardiogram may show signs suggestive of 
endomyocardial fibrosis, such as areas of endocardial fibrosis, ventricular diastolic restriction, and amputation of the tip of the affected ventricular cavity.

On coronary angiography, obliteration of the right and left ventricular cavities have been reported, as has the presence of tricuspid or mitral insufficiency, or both, with amputation of the tip of the affected ventricle. These findings were present in our case, especially in the left ventricle.

The anatomicopathological study of endomyocardial fibrosis shows some major findings as follows: obliteration of the trabeculae of the inflow tract and body of the right or left ventricle, or both, by fibrous tissue containing neoformed vessels and disordered elastic fibers. Myocardial impairment occurs with involvement of the mitral and tricuspid valvular apparatus ${ }^{4}$. These aspects could be observed in our case, in which diffuse fibrous endocardial thickening was present with an important functional repercussion in the mitral valve.
The differential diagnosis with endocardial fibroelastosis, a restrictive disorder more frequent in the age group of our patient ${ }^{11,12}$, was based both on the gross appearance and on the histological characteristics of the lesion. In fibroelastosis, the limit between the thickened endocardium and the myocardium is very clear ${ }^{11,12}$, which was not observed in our patient, who had fibrotic bundles infiltrating the myocardium. In endomyocardial fibrosis, endocardial inflammation not necessarily rich in eosinophils is characteristic, in addition to neoformed vessels ${ }^{10}$, as found in our patient. Some authors have cogitated that characteristics, such as fibrosis, inflammation, and neoformed vessels, may imply a possible organization of endocardial thrombi in the pathogenesis of the lesion. Another aspect that allows the differentiation between the 2 entities is the arrangement of the bundles of elastic fibers, which is parallel in fibroelastosis and disordered in endomyocardial fibrosis.

\section{References}

1. Davies JNP, Ball JD. The pathology of endomyocardial fibrosis in Uganda. $\mathrm{Br}$ Heart J 1995; 17: 337 .

2. Ball JD, Williams AW, Davies JNP. Endomiocardial fibrosis. Lancet 1959; i: 1049

3. Mandroi W, Vieira SR, Hemb R, Freitas FM, Azevedo DF, Faraco EZ. Achados hemodinâmicos e cineangiocardiográficos na fibrose endomiocárdica. Arq Bras Cardiol 1982; 38: 173-80.

4. Barreto ACP, Luz PL, Oliveira AS, et al. Determinants of survival in endomyocardial fibrosis. Circulation 1989; 80: 1177-82.

5. Jagob JLB, Garzon SAC, Silveira LC, Lorga AM. Fibrose endomiocárdica em criança de 2 anos de idade. Relato de caso. Arq Bras Cardiol 1988; 50: 413-6.

6. Guimarães AC, Esteves JP, Filho AS, Macedo V. Clinical aspects of endomyocardiofibrosis in Bahia, Brazil. Am Heart J 1971; 81: 7-19.
7. Funder JW. Steroids, hypertension and cardiac fibrosis. Blood Press Suppl 1995; $39-42$.

8. Mayet J, Kanagaratnam P, Lincoln C, Oldershaw P. Hipereosinophilic syndrome: endomyocardial fibrosis. Heart 1997; 77: 391.

9. Chan L, Chiang BN, Kong CW, Lee JB, Wang SP, Hsu TL. Endomyocardial fibrosis with massive endocardial calcific depositis. Clin Cardiol 1987; 10: $541-5$.

10. Santos CL, Moraes CR, Santos FL, Moraes F, Brindeiro Filho D. Endomyocardial fibrosis in children. Cardiol Young 2001; 11: 205-9.

11. Ângelo A, Kulova A, Gurdevsky. Endocardial fibroelastosis. Clinico-patholgical study of 38 cases. Pathol Res Pract 1984; 178: 84- 8 .

12. Venugopalan P, Stewart JM. Endocardial fibroelastosis. Med J 2001; 2: 1-23. 